\title{
CHARACTERIZATION OF BIOACTIVE RESTORATION/DENTINE INTERFACE
}

\author{
Hamdi Hosni Hamdan Hamama*
}

\begin{abstract}
Objectives: To characterize the bonding interfaces of a calcium silicate-based restorative material (Biodentine) and CPP/ACP-modified glass ionomer with dentine. Also, this study was designed to evaluate the influence of dentine-surface treatment with poly acrylic acid on the bonding of both materials to dentine.
\end{abstract}

Methods: Sixty caries-free human molars were used in this study. Specimens were randomly divided into three groups according to type of restorative material used $(n=20)$; calcium silicatebased (Biodentine, Septodont, France), conventional GIC Fuji VII-EP, GC Corporation, Tokyo, Japan) or CPP/ACP-modified glass ionomer (Fuji VII-EP, GC Corporation, Tokyo, Japan). Each group was divided into 2 sub-groups relative to the dentine surface treatment method $(n=10)$; the dentine in control group was left without treatment, while the dentine surface in test groups was conditioned with poly acrylic acid (PAA) for 10 s prior to the application of restorative. In each subgroup, 5 specimens were double-labeled with fluorescein and rhodamine-B dyes then observed under a confocal laser scanning microscopy (CLSM). While the remaining 5 specimens were observed under scanning electron microscopy (SEM) then subjected to EDX elemental analysis.

Results: CLSM micrographs showed that the mineral rich zone (MRZ) was obvious in nonconditioned Biodentine groups compared to conditioned groups. Both GICs groups exhibited obvious ion-exchange zones. In the non-conditioned Biodentine groups, polygonal crystal deposition was noticed beneath the hybrid layer. These findings were confirmed by the outcome of micromorphological analysis of restoration/tooth interface using SEM.

Conclusions: Calcium silicate-based restorative material has a great affinity to exchange ions with tooth substrate and to bond chemically with dentine. The results of this study showed that calcium silicate-based restorative material (Biodentine) can be applied directly on dentine surface without any pre-surface treatment.

KEYWORDS: Bioactive restorations, Biodentine, Calcium Silicate, CPP/ACP, Glass Ionomer, Confocal Laser Scanning Microscopy

\footnotetext{
* Lecturer, Operative Dentistry Department, Faculty of Dentistry, Mansoura University
} 


\section{INTRODUCTION}

Creation of hermetically-sealed restorations is one of the ultimate goals of restorative dentistry. Recent minimally invasive dentistry concepts promote maximum preservation of natural tooth substrate. This can be achieved by using current innovative caries removal methods and application of recent adhesive dentistry concepts. The outcome of several evidence-based studies $^{1-3}$ revaeled that dental adhesive joints are considered as the weak link between restorative materials and tooth substrate. Failures in tooth/restoration interface may lead to formation of marginal gaps and consequently allows the microorganisms, toxins and food debris to occupy these negative spaces. Many attempts were performed to overcome this problem and one of the most successful trials was using of bioactive restorative materials. ${ }^{4}$

The in-situ bio-remineralization is a novel minimally invasive remineralization treatment that has been recently introduced into the medical field, particularly in orthopedics. Bio-remineralization is defined as a physicochemical process depends on an induced crystallization of calcium and phosphorus ions under well-controlled conditions. ${ }^{5}$ The material which has the capacity to stimulate biological response from the body (e.g. tissue bonding) is referred to as bioactive material. ${ }^{6}$ This category of materials can influence a response from living tissue or organisms such as inducing formation of hydroxyapatite. Several bioactive materials were recently introduced into the field of dentistry; mineral trioxide aggregate (MTA), Tri-calcium silicates, bioactive glass and new generations of glass ionomer cements (GIC).

In 1960, calcium silicate cements were introduced to the dental field as the first tooth-coloured restorative material. In the past two decades, calcium silicate-based restorative materials were significantly improved, and many bioactive derivatives were generated. This category of restorative materials exhibits an excellent biocompatibility, as well as, it can bond (chemically) to tooth substrate. It has been widely used in the field of endodontics, particularly in vital pulp therapy and repair of perforations. The popular example of this group is MTA which exhibited excellent antibacterial and bioremineralization activities. ${ }^{7}$ On the other hand, MTA suffer from long-setting time (24h under wet condition) which limits it use in the field of restorative dentistry. Few years ago, a new formula of fast-set tricalcium silicate-based restorative materials was introduced to overcome MTA drawback. Several studies ${ }^{8-10}$ were conducted to evaluate the efficiency of this new bioactive restorative material. The outcome of these studies revealed that the new bioactive restorative formula demonstrated a significant remineralization potential and could be considered as an alternative to traditional cavity base and liners. ${ }^{10}$ The outcome of micro-morphological observations of a previous study showed a clear evidence of calcium ion deposition 'crystallization' within the hybrid-like layer at restoration/tooth. ${ }^{11}$ Conversely, some studies reported that mechanical properties and remineralization potential of such bioactive restorative materials are still questionable. ${ }^{12,13}$ Also, these studies recommended further micromorphological analysis for the formed hybrid-like layers using advanced technologies.

The conventional glass ionomer was introduced in 1972 and since this date several attempts were conducted to improve its original chemical formula. These trials including addition of hydroxyapatite, metal, resins and bioactive ingredients to the original glass powder. One of the recent GIC modifications is incorporation of casein phosphopeptide / amorphous calcium phosphate (CPP/ACP) to the original formula. This may enhance the bioactivity and anticariogenic effect of the restorative material. ${ }^{14}$ Until present, there is few information about bonding nature of this bioactive glass ionomer to dentine.

In light of above, this study was conducted to comprehensively evaluate the micromphological and chemical changes of bioactive restorative materials/dentine interfaces using advanced characterization methodologies. These methods include 
double-labeling of restoration/tooth interface under confocal laser scanning microscopy (CSLM) and elemental analysis of hybrid-like layer using an energy dispersive $\mathrm{x}$-ray (EDX) mapping. Also, this study was designed to evaluate the influence of dentine-surface treatment with poly acrylic acid on the bonding of both materials to dentine.

\section{MATERIALS AND METHODS}

This study was approved by the Institutional Review Board of the University of Hong Kong (IRB ref no: UW 17-070) for the collection of teeth. Commercially available calcium silicate and CPP/ACP-modified GIC restorative materials were used in this study (Table 1).

TABLE (1) Materials used in the study

\begin{tabular}{|c|c|c|}
\hline \multirow{2}{*}{ Biodentine } & Ingredients & Company \\
\hline & Calcium silicate & Septodont, France \\
\hline 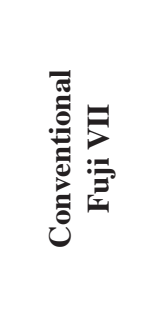 & $\begin{array}{c}\text { Powder: } \\
\text { Fluoro-alumino silicate } \\
\text { glass } 95-99 \% \\
\text { CPP-CAP 1 - 5\% } \\
\text { Pigment Trace } \\
\text { Liquid: } \\
\text { Distilled water 50\% } \\
\text { Polyacrylic acid } 40 \%\end{array}$ & \multirow{2}{*}{$\begin{array}{c}\text { GC } \\
\text { Corporation,Tokyo, } \\
\text { Japan }\end{array}$} \\
\hline 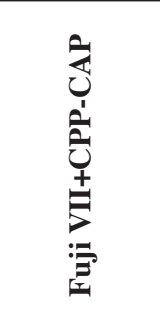 & $\begin{array}{c}\text { Powder: } \\
\text { Fluoro-alumino silicate } \\
\text { glass } 95-99 \% \\
\text { CPP-CAP 1 - 5\% } \\
\text { Pigment Trace } \\
\text { Liquid: } \\
\text { Distilled water 50\% } \\
\text { Polyacrylic acid 40\% }\end{array}$ & \\
\hline
\end{tabular}

\section{Study design and specimen preparation}

Sixty sound 'caries-free' human molars were used. The teeth were stored in $0.5 \%$ chloramine $\mathrm{T}$ solution at $4^{\circ} \mathrm{C}$ and used within six months following extraction. The occlusal enamel was removed using a low-speed diamond saw (Isomet, Buehler, USA). Exposed dentine samples were randomly divided into 3 groups according to type of restorative material used $(\mathrm{n}=20)$; calcium silicate- based (Biodentine, Septodont, France), conventional GIC Fuji VII-EP, GC Corporation, Tokyo, Japan) or CPP/ACP-modified glass ionomer (Fuji VII-EP, GC Corporation, Tokyo, Japan). Each group was divided into 2 sub-groups according to the dentine surface treatment method $(n=10)$; the dentine in control group was left without treatment, while the dentine surface in test groups was conditioned with poly acrylic acid (PAA) for 10s prior to the application of restorative materials. In each subgroup, 5 were double-labeled and observed under a confocal laser scanning microscopy (CLSM). While the remaining 5 specimens were observed under scanning electron microscopy (SEM) then subjected to EDX elemental analysis.

\section{Double-labeling of restoration/tooth interface and observation under CSLM}

Two environmentally safe florescent labeling dyes were used in this study; fluorescein and rhodamine-B. The fluorescein dye was the marker of restorative materials and was incorporated to the unset materials prior to its application. Following the application of the restorative material, teeth were received a horizontal cut through the cementoenamel junction, which divided the pulp chamber into coronal and radicular compartments. Only the coronal portion was used in this study to act as a reservoir to rhodamine-B labeling solution. The coronal compartment was placed in upside down position and the pulp chamber reservoir was filled with rhodamine-B solution and left for $3 \mathrm{~h}$ (Fig.1). Then the dye was copiously rinsed with distilled water. Each restored 'labeled' specimen was vertically sectioned into 2 halves through the restoration center using a low-speed diamond saw (Isomet, Buehler, USA) (Fig 1). The cut surface was wet polished with (600-, 800-, 1200-, 2400and 4000- grit) silicon carbide papers (Microcut ${ }^{\mathrm{TM}}$, Buehler,Lake Bluff, IL, USA) respectively, then followed by lapping with a polishing cloth using 6, 3,1 $\mu \mathrm{m}$ diamond pastes (Diamat, Pace Technologies, Tuscon, AZ, USA). Specimens were ultrasonically cleaned in distilled water. The Observations were performed using confocal 


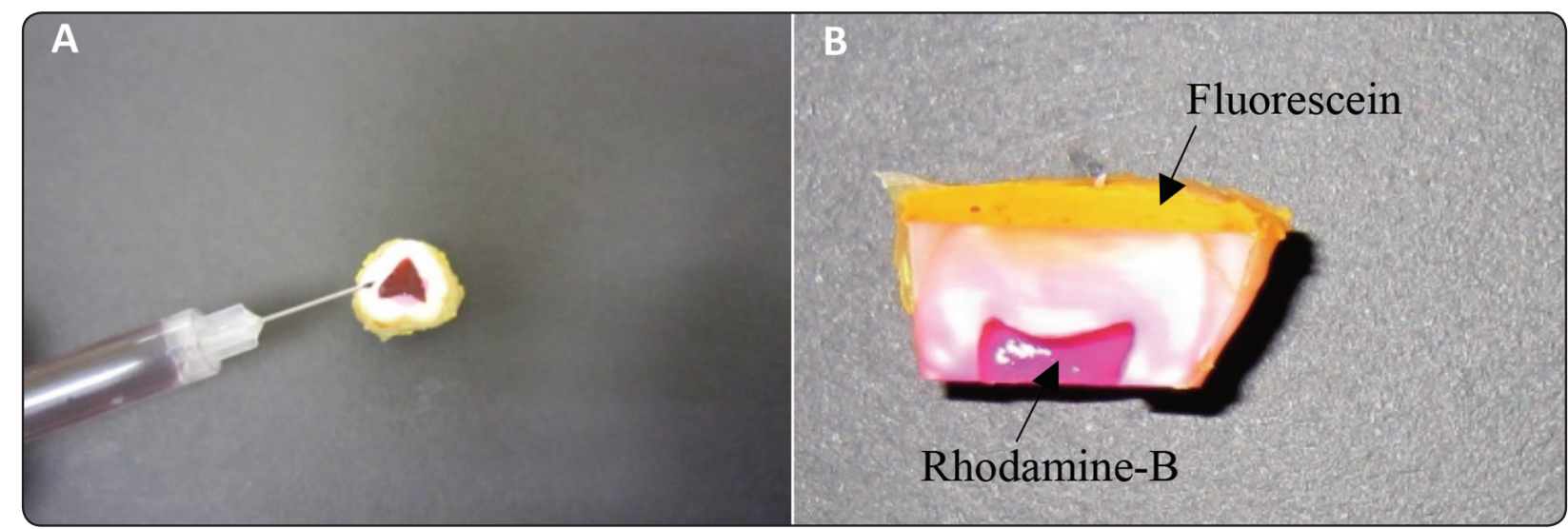

Fig. (1) Showing procedures of double-labeling of the restoration/tooth interface. A: Pulp chamber reservoir filled with rhodamine-B solution

laser scanning microscopy (CLSM) (Fluoview 1000 , Olympus, PA, USA). In order to reduce observation bias, the (hi-lo) grey color mode was initially used to mark the over-saturated dye zones.

\section{Elemental analysis of restoration/tooth interface using EDX mapping}

The previously mentioned study design was followed in conducting this test. The enamel was removed, and restorative materials were applied on the freshly-cut dentine surface. The bonded specimens were vertically sectioned through the restoration center into two halves. The cut surface was wet polished with (600-, 800-, 1200-, 2400and 4000- grit) silicon carbide papers (Microcut ${ }^{\mathrm{TM}}$, Buehler,Lake Bluff, IL, USA) respectively, then followed by lapping with a polishing cloth using 6, 3, $1 \mu \mathrm{m}$ diamond pastes (Diamat, Pace Technologies, Tuscon, AZ, USA). Specimens were ultrasonically cleaned in distilled water. The specimens, which utilized in this test, were not coated. The resin/dentine interface was subjected to EDX analysis with EDX software attached to a field emission scanning electron microscopy. The restorative elemental marker for each restoration was as follow: Aluminum for GIC groups and silica for Biodentine groups.

\section{Micromorphological analysis of restoration/ tooth interface under SEM}

The same specimen preparation procedures of elemental analysis test were followed. Resin/dentine interface was subjected to an acid-base challenge using $10 \%$ orthophosphoric acid solution for $5 \mathrm{~s}$, followed by application of $5.5 \%$ sodium hypochlorite solution for $5 \mathrm{~m}$. The observing surface was gold-sputter coated and observed under scanning electron microscopy in secondary electron mode.

\section{RESULTS}

\section{Double-labeling of restoration/tooth interface and observation under CSLM}

The CSLM micrographs of the restoration/ tooth interface are presented in figure 2. Confocal scanning laser microscopy micrographs revealed that both GICs groups exhibited obvious iondiffusion zones (Fig 2-A and 2-B). In GIC groups, the ion-exchange zones were clearly observed in the conditioned groups in comparison with the corresponding non-conditioned groups (Fig 2-C and 2-D). An obvious mineral-rich zone (MRZ) was observed within all calcium silicate-based restorative material (Biodentine) groups (Fig 2-A and 2-B). Moreover, the ion-exchange zone was obvious in the non-conditioned Biodentine groups compared to conditioned ones (Fig 2-A). 


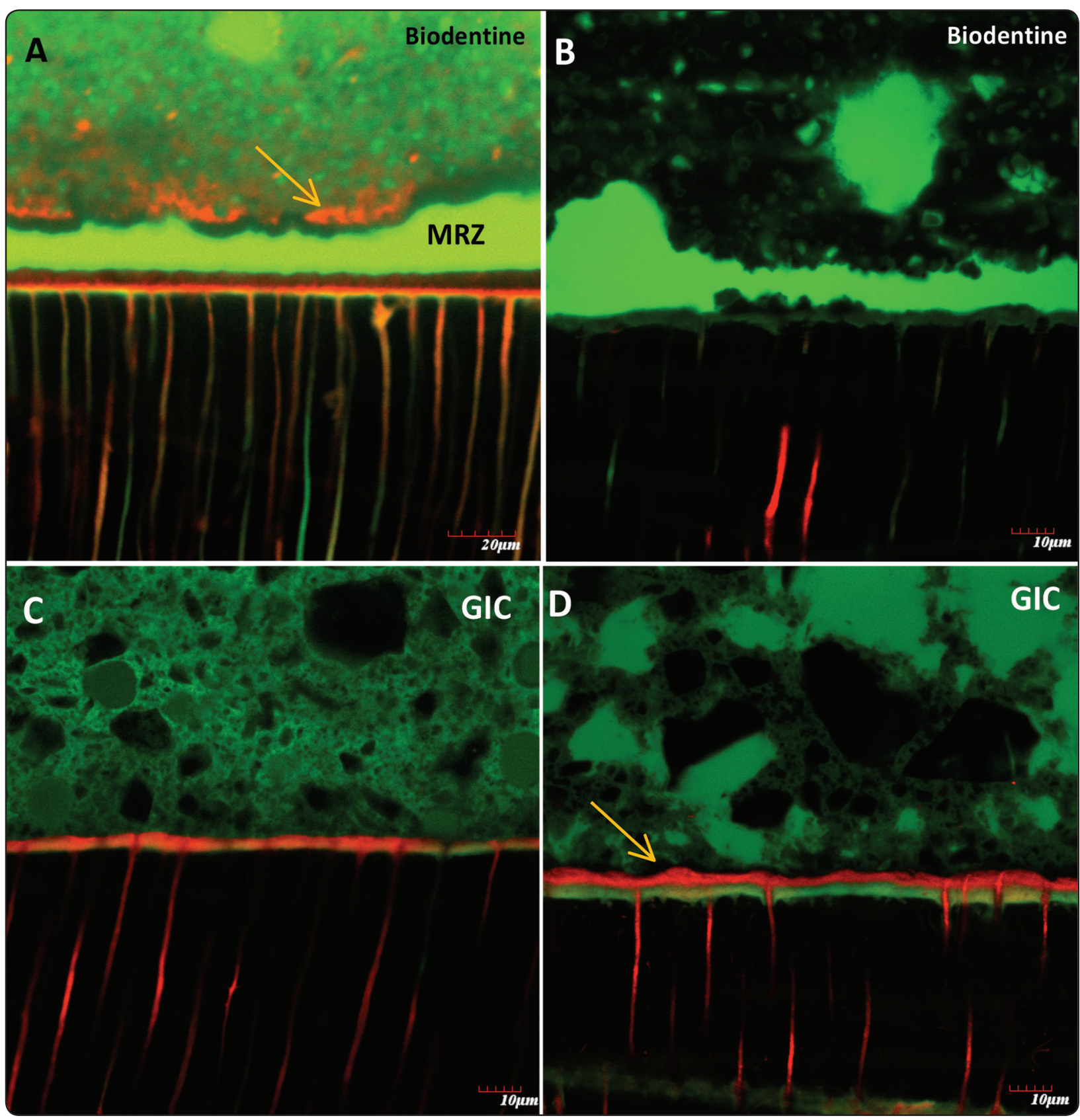

Fig. (2) CSLM micrographs of restoration/dentine interface. A: Non-conditioned Biodentine group exhibited a distinct ionexchange zone with obvious mineral rich zone (MRZ) above the hybrid-like layer. B: Conditioned Biodentine group; MRZ is noticeable in conditioned Biodentine group, however, the ion-exchange zone is less observable. C: Non-conditioned GIC groups (either conventional or CPP/ACP-modified GICs) showed less obvious ion-exchange zones. D: Conditioned GIC groups showed obvious ion-exchange zone in comparison to non-conditioned GICs groups. 


\section{Elemental analysis of restoration/tooth interface using EDX mapping}

EDX mapping showed obvious ion-exchange at restoration/interface (hybrid-like layer). In GICs groups the aluminum (GIC marker) exhibited a uniform ion exchange pattern with a clear evidence of diffusion through the hybrid-like layer. Also, in calcium-silicate groups EDX maps showed distinct zones of ion-exchange of silica particles within restoration/tooth interface. Furthermore, EDX showed characteristic polygonal crystal deposition on the dentine surface (Fig.3)

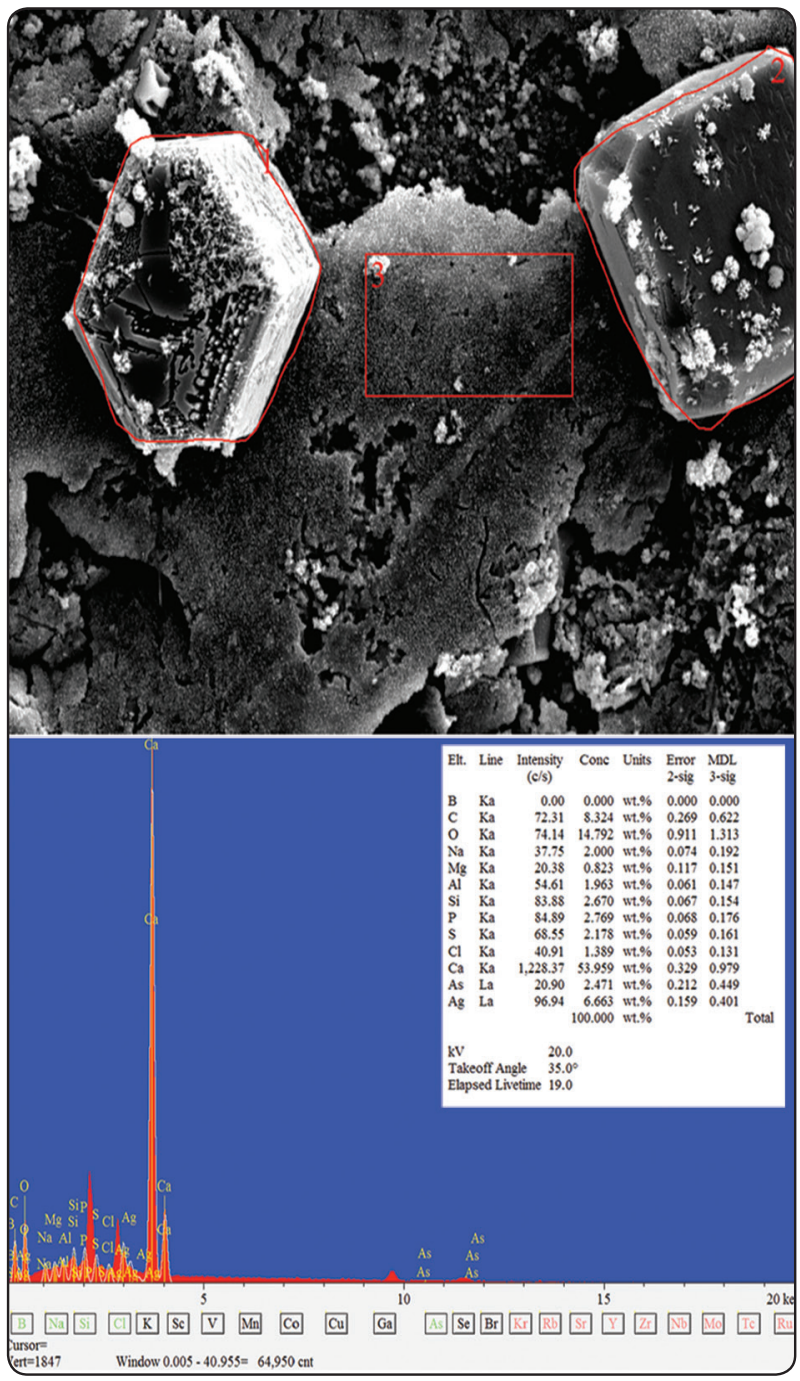

Fig. (3) EDX micrographs and elemental reports showing a unique polygonal crystallization pattern

\section{Micromorphological analysis of restoration/ tooth interface under SEM}

The micromorphological analysis of restoration/ tooth interface showed a typical formation of acidbase resistant hybrid-like layer in both conditioned GIC materials. Scanning electron micrographs of tri-calcium silicate groups showed a thick acid-base resistant hybrid like-layer with distinct crystallization pattern (Fig 4)

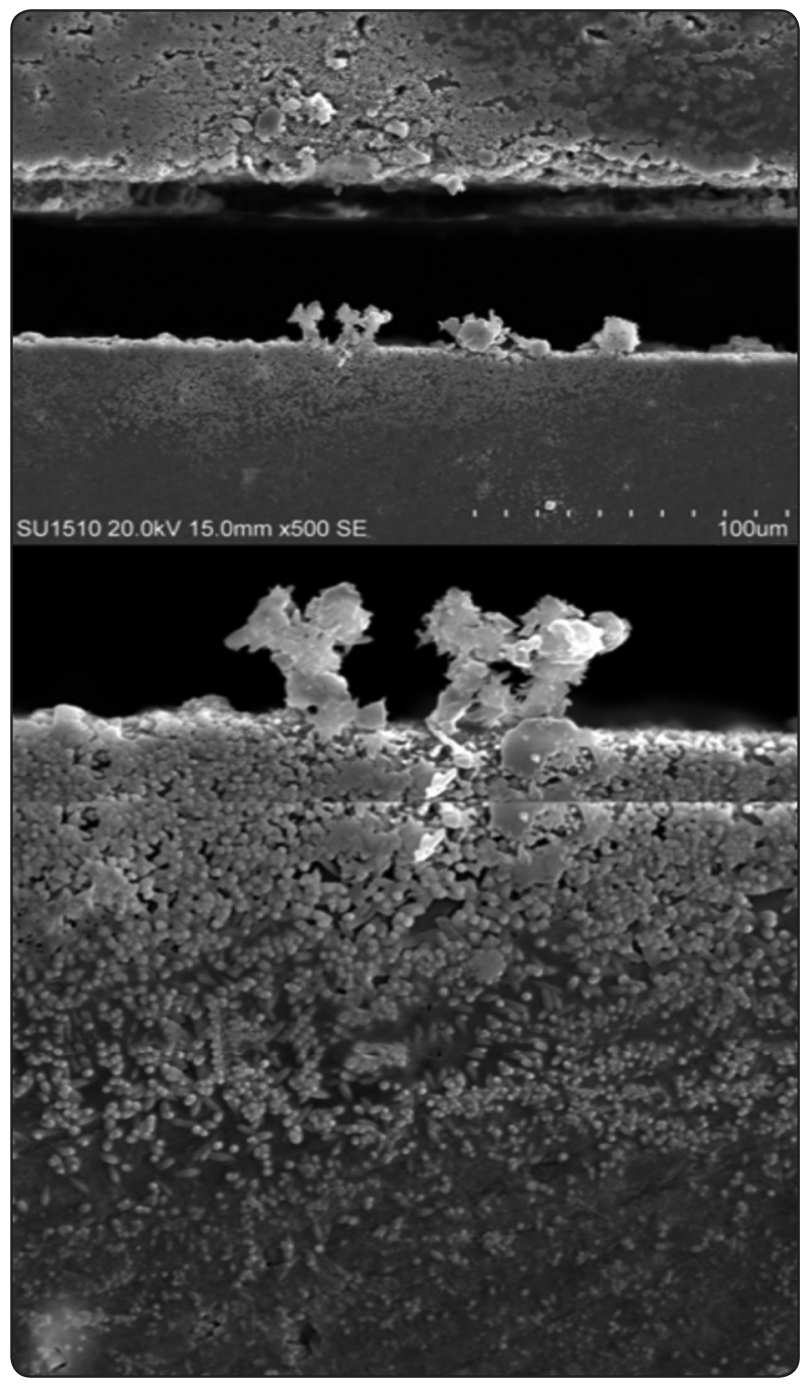

Fig. (4) SEM micrographs showing deposition of acid-base crystals at hybrid-like layer level. 


\section{DISCUSSION}

Bio-remineralization of caries-affected dentine becomes an important topic of interest in the field of restorative dentistry. Thus, studying the micromorphological characteristics of restoration/ tooth interface improves our understanding of the bonding characteristics of any restorative material. In this study, a new calcium silicate-based material, claimed to be bioactive, was selected. This material exhibited a relatively fast-setting time compared to conventional MTA, which required at least $24 \mathrm{~h}$ for complete setting. The manufacturer of this material claimed its bioactivity and great bonding affinity, hence, a conventional GIC was selected to act as control group. Also, a CPP/ACP-modified type of GICs was used in order to evaluate the benefits of incorporation of $\mathrm{CPP} / \mathrm{ACP}$ into conventional GIC powder.

There commonly used method for characterization of restoration/tooth interface is observation under a scanning electron microscopy. Although, the outcome of this traditional observation method provides useful information about the formed hybrid layer, there are several challenges may influence its accuracy, particularly when used for evaluation cements. The water content of the cement when rapidly evaporate inside SEM vacuum chambers creates microcracks and debonding of the restoration/tooth interface. Also, this traditional method can not provide accurate information about the true ion-exchange between the restorative material and the dentine. Therefore, in the current study doublelabelling and EDX-mapping were used. In order to reduce the crack formation within restorative material inside SEM chamber, an environmental SEM was used.

The outcome of double-labeling of restoration/ tooth interface test showed that ion-exchange zone was clearly observed in all conditioned GIC groups. This finding is in agreement with a previous study by Hamama et.al ${ }^{15}$ which showed that chemical bonding of GIC enhanced by conditioning of the dentine surface with PAA prior to the application of the GIC restorative material. The results of this study showed no significance between CPP/ACPmodified GIC and conventional GICs. This is attributed to the great similarity of the ingredients of the powder small portion of the incorporated $\mathrm{CPP} / \mathrm{ACP}$ which resemble $1 \%$ of the total powder content. Although, this small percentage of $\mathrm{CPP} /$ ACP does not influence the results, it was detectable in EDX maps. Accordingly, this slight increase in $\mathrm{Ca}$ and $\mathrm{P}$ ions may be beneficial in remineralization of caries-affected dentine.

In calcium silicate (Biodentine) groups, mineral-rich zones were observed, and this can be considered as a sign of bioactivity. Moreover, EDX mapping showed a unique polygonal crystal formation on dentine surface. These crystals formed by combination of calcium-phospho-silicate and the outcome of micromorphological analysis revealed that these crystals exhibited an acid-based resistant nature (Fig 4). Hence, these crystals may reduce the incidence of caries recurrence and strength the compromised caries-affected dentine. The bio reactivity of tri-calcium silicate may be attributed to the presence of water in the dentine. Thus, this procedure is continuous and is not limited to the material setting period.

Nevertheless, the manufacturer of the new calcium silicate-based material (Biodentine) recommended applying the restorative material directly without dentine surface treatment, in the current study we tried to evaluate the effect of conditioning the dentine surface with poly acrylic acid for $10 \mathrm{~s}$. Thus, the assumption of the current study was that conditioning of the dentine surface might modify the smear layer and consequently enhance the bonding between tri-calcium silicatebased restoration to dentine. However, in light of the results of this study, this assumption was no longer accepted. Confocal micrographs revealed that nonconditioned Biodentine group showed a clear ionexchange zone in comparison with conditioned 
group. This also was confirmed by SEM images which showed a distinct crystallization formation at hybrid layer level. Hence, the application of conditioner may disturb the maturation of the crystal on dentine surface.

\section{CONCLUSIONS}

Calcium silicate-based restorative material has a great affinity to exchange ions with tooth substrate and to bond chemically with dentine. The results of this study showed that calcium silicate-based restorative material (Biodentine) can be applied directly on dentine surface without any pre-surface treatment.

\section{ACKNOWLEDGMENT}

The author thank technicians of Hard Tissue and Oral Bio-sciences Laboratories, Faculty of Dentistry, The University of Hong Kong for their great help in preparation and imaging the specimens.

\section{REFERENCES}

1. Mak YF, Lai SC, Cheung GS, Chan AW, Tay FR, Pashley DH Micro-tensile bond testing of resin cements to dentin and an indirect resin composite. Dent Mater 2002;18:609-621.

2. Zhang Z, Beitzel D, Mutluay M, Tay FR, Pashley DH, Arola D. On the durability of resin-dentin bonds: Identifying the weakest links. Dent Mater 2015;31:1109-1118.

3. Yang B, Adelung R, Ludwig K, Bossmann K, Pashley DH, Kern M. Effect of structural change of collagen fibrils on the durability of dentin bonding. Biomaterials 2005;26:5021-5031.

4. Cheng L, Zhang K, Zhang N, et al. Developing a New Generation of Antimicrobial and Bioactive Dental Resins. J Dent Res 2017;96:855-863.
5. Zhang K, Zhang N, Weir MD, Reynolds MA, Bai Y, Xu HHK. Bioactive Dental Composites and Bonding Agents Having Remineralizing and Antibacterial Characteristics. Dent Clin North Am 2017;61:669-687.

6. Jones JR, Boccaccini AR. A forecast of the future for biomaterials. J Mater Sci Mater Med 2006;17:963-964.

7. Torabinejad M, Parirokh M, Dummer PMH. Mineral trioxide aggregate and other bioactive endodontic cements: an updated overview - part II: other clinical applications and complications. Int Endod J 2018;51:284-317.

8. Malkondu O, Karapinar Kazandag M, Kazazoglu E. A review on biodentine, a contemporary dentine replacement and repair material. Biomed Res Int 2014;2014:160951.

9. Agrafioti A, Tzimpoulas N, Chatzitheodoridis E, Kontakiotis EG. Comparative evaluation of sealing ability and microstructure of MTA and Biodentine after exposure to different environments. Clin Oral Investig 2016;20:15351540 .

10. Kim JR, Nosrat A, Fouad AF. Interfacial characteristics of Biodentine and MTA with dentine in simulated body fluid. J Dent 2015;43:241-247.

11. Hamama H, Yiu C, Burrow M. Bonding of Calcium Silicate-based Restorative Material to Dentine. J Dent Res 2017;96

12. Rajasekharan S, Martens LC, Cauwels RG, Verbeeck RM Biodentine material characteristics and clinical applications: a review of the literature. Eur Arch Paediatr Dent 2014;15:147-158.

13. Camilleri J. Investigation of Biodentine as dentine replacement material. J Dent 2013;41:600-610.

14. Koizumi H, Hamama HH, Burrow MF. Evaluation of adhesion of a CPP-ACP modified GIC to enamel, sound dentine, and caries-affected dentine. International Journal of Adhesion and Adhesives 2016;66:176-181.

15. Hamama HH, Burrow MF, Yiu C. Effect of dentine conditioning on adhesion of resin-modified glass ionomer adhesives. Aust Dent J 2014;59:193-200. 